\title{
The efficacy of daily chlorhexidine bathing for preventing healthcare-associated infections in adult intensive care units
}

\author{
Hua-ping Huang ${ }^{1}$, Bin Chen ${ }^{2}$, Hai-Yan Wang ${ }^{1}$, and $\mathrm{Me} \mathrm{He}^{1}$
}

${ }^{1}$ Nursing Administration, ${ }^{2}$ Intensive Care Unit, Mianyang Central

Hospital, Mianyang, China

Received: July 24, 2015

Revised : August 23, 2015

Accepted: September 6, 2015

\section{Correspondence to}

Hua-ping Huang, R.N.

Nursing Administration, Mianyang Central Hospital,

No. 12, Changjia Alley, Jing-

zhong Street, Fucheng District,

Mianyang 621000, China

Tel: +86-816-223-9671

Fax: +86-816-222-2566

E-mail:jrzhou26@aliyun.com
Background/Aims: Healthcare-associated infections (HAIs) in critically ill patients with prolonged length of hospital stay and increased medical costs. The aim of this study is to assess whether daily chlorhexidine gluconate (CHG) bathing will significantly reduce the rates of HAIs in adult intensive care units (ICUs). Methods: PubMed, EMBASE, and the Cochrane Central Register of Controlled Trials were systematically searched until December 31, 2014 to identify relevant studies. Two authors independently reviewed and extracted data from included studies. All data was analyzed by Review Manager version 5.3.

Results: Fifteen studies including three randomized controlled trials and 12 quasi-experimental studies were available in this study. The outcomes showed that daily CHG bathing were associated with significant reduction in the rates of primary outcomes: catheter-related bloodstream infection (risk ratio [RR], 0.44; 95\% confidence interval [CI], 0.32 to $0.63 ; p<0.00001$ ), catheter-associated urinary tract infection (RR, 0.68 ; $95 \% \mathrm{CI}, 0.52$ to $0.88 ; p=0.004$ ), ventilator-associated pneumonia (RR, $0.73 ; 95 \% \mathrm{CI}, 0.57$ to $0.93 ; p=0.01$ ), acquisition of methicillin-resistant Staphylococcus aureus (RR, 0.78; 95\% CI, 0.68 to $0.91 ; p=0.001$ ) and vancomycin-resistant Enterococcus (RR, 0.56; $95 \%$ CI, 0.31 to $0.99 ; p=0.05$ ).

Conclusions: Our study suggests that the use of daily CHG bathing can significantly prevent HAIs in ICUs. However, more well-designed studies are needed to confirm these findings.

Keywords: Chlorhexidine gluconate; Bathing; Infection; Intensive care units

\section{INTRODUCTION}

Healthcare-associated infections (HAIs) are the most common cause of morbidity and mortality among hospitalized patients. In 2011, approximately 648,000 patients experienced 721,800 HAIs in United States acute care hospitals [1]. Critically ill patients in intensive care units (ICUs) are at high risk for infection as a result of underlying immunodeficiency; comorbidity; and placement of invasive devices, such as endotracheal tubes and intravascular devices [2]. Infections are strongly associated with prolonged length of stay (LOS) and increased medical charges [3].

Healthcare professionals have proposed several strategies for preventing HAIs, including compliance with hand hygiene, aseptic technique, and contact isolation precautions for patients, but these strategies can be difficult to maintain. Chlorhexidine gluconate $(\mathrm{CHG})$ is a widely used antiseptic agent that has excellent antimicrobial activity and rapidity of action [4]. Furthermore, 
in contrast with other antiseptic agents, the residual antimicrobial activity of CHG is not affected by the presence of body fluids and blood [5]. Some previous studies have demonstrated that daily CHG body bathing can effectively prevent HAIs, such as catheter-related bloodstream infections (CRBSIs) [6-12], surgical site infections [13], and the colonization of multidrug-resistant organisms (MDROs) $[10,12,14]$. However, the findings from a recent single-center, cluster-randomized controlled trial (RCT) challenged this approach [15], reporting that daily CHG bathing did not reduce rates of infection-related primary outcomes when compared with routine care (rate difference, -0.04; 95\% confidence interval [CI], -1.10 to $1.01 ; p=0.95)$. To solve this ongoing issue, we performed this systematic review and meta-analysis to assess whether daily CHG bathing, compared with usual care, significantly decreases the rates of HAIs in adult ICUs.

\section{METHODS}

We followed the Preferred Reporting Items for Systematic Reviews and Meta-analyses (PRISMA) statement to report this systematic review and meta-analysis [16]. The protocol of this study was registered on PROSPERO (the international register of systematic reviews; registration number: CRD42014014973).

\section{Search strategy}

The searches of PubMed, EMBASE, and the Cochrane Central Register of Controlled Trials (CENTRAL) were performed from their inception to December 31, 2014. The searches were restricted to English publications and human subjects. The following search terms were used: chlorhexidine, body wash, bathing, showering, hospital-acquired infection, methicillin-resistant Staphylococcus aureus (MRSA) or vancomycin-resistant Enterococcus (VRE) colonization/acquisition, ICU, and critically ill patients. The reference lists of the original and related reviews were also manually searched to identify any additional relevant studies. The latest search was performed on March 31, 2015.

\section{Study selection}

All included studies had to meet the following criteria:
(1) participants: adult patients in ICUs; (2) intervention: use of daily CHG bathing. If patients were treated with CHG washcloths, we defined it as daily CHG bathing; (3) comparison: soap and water or other routine care; (4) outcomes: at least one of the quantitative outcomes mentioned in the next section of this article was reported; and (5) study design: RCTs, interrupted time series studies, and before and after studies. Studies were excluded if they combined CHG bathing with oral or topical decontamination; if participants were in pediatric ICUs, general wards, cancer wards, or nursing homes; or if they were protocols, unpublished or duplicated studies, editorials, or review articles.

\section{Data extraction}

Two of the authors (HPH and BC) independently extracted and summarized the data from each included study. Any disagreement was resolved by discussion and consensus. The following characteristics of the studies were extracted: the first author, year of publication, country, study design, setting, patient characteristics, intervention protocols, and outcomes.

The primary outcomes included CRBSI, catheter-associated urinary tract infection (CAUTI), ventilator-associated pneumonia (VAP), and acquisition of MRSA and VRE. The secondary outcomes were LOS, overall hospital mortality, and adverse events.

\section{Quality assessment}

Two of the authors (HPH and HYW) independently used the Cochrane Risk of Bias Tool to assign a judgment of low, unclear, or high risk of bias for RCTs according to the following items: random sequence generation, allocation concealment, blinding of participants and personnel, blinding of outcome assessment, incomplete data, selective reporting, and other bias [17]. The Newcastle-Ottawa Quality Assessment Scale was used to assess the methodological quality of non-randomized studies, which consist of three items: selection, comparability, and outcome assessment [18]. A study can be awarded a maximum of one star for each numbered item within the selection and outcome categories. A maximum of two stars can be given for comparability. A score of 6 or more indicates a study with high quality. 


\section{Statistical analysis}

Review Manager Software version 5.3 (Cochrane Collaboration, Oxford, United Kingdom) was used to pool data. Differences between the two groups were presented as a weighted mean difference (WMD) with a 95\% CI for continuous outcomes and risk ratio (RR) with a 95\% CI for dichotomous outcomes. Cochrane's Q test and the $I^{2}$ statistic were used to assess heterogeneity between studies, which was defined as a $p$ value for Cochran's Q test of $<0.1$ and an $I^{2}$ value of > 50\% [19]. If there was significant heterogeneity, the random effects model was used to combine the data; otherwise, the fixed-effects model was used. A $p<0.05$ was judged as statistically significant.

\section{RESULTS}

\section{Study selection}

Fig. 1 shows a diagram of the study selection process. Three hundred and thirty-nine relevant publications were identified during the initial search. Fifteen studies, including three RCTs $[6,12,15]$ and 12 quasi-experimental studies [7-11,14,20-25], met the criteria and were included in the final meta-analysis.

\section{Characteristics of included studies}

Table 1 presents the characteristics of each included study. All included studies were conducted after 2005 in the United States, except one study in France [25] and one in Mexico [22]. Seven studies occurred in mixed ICUs $[7,8,12,15,21,22,24]$, four in medical ICUs $[6,11,14,25]$, two in surgical ICUs [9,20], one in a general ICU [23], and one in a trauma ICU [10]. For the intervention protocol of the included studies, all studies used 2\% CHG-impregnated cloths for bathing except for one that used $4 \%$ CHG-based soap [24].

\section{Methodological quality assessment}

The risk of bias assessment summaries of the included studies are presented in Tables 2 and 3. For RCTs, only one study adequately reported the sequence generation method [15]. No studies provided the details about allocation concealment or the blinding methods. All of the studies used the intention-to-treat analysis to handle the missing data. Overall, all of the included RCTs were judged as having a high risk of bias. The risk of bias as-

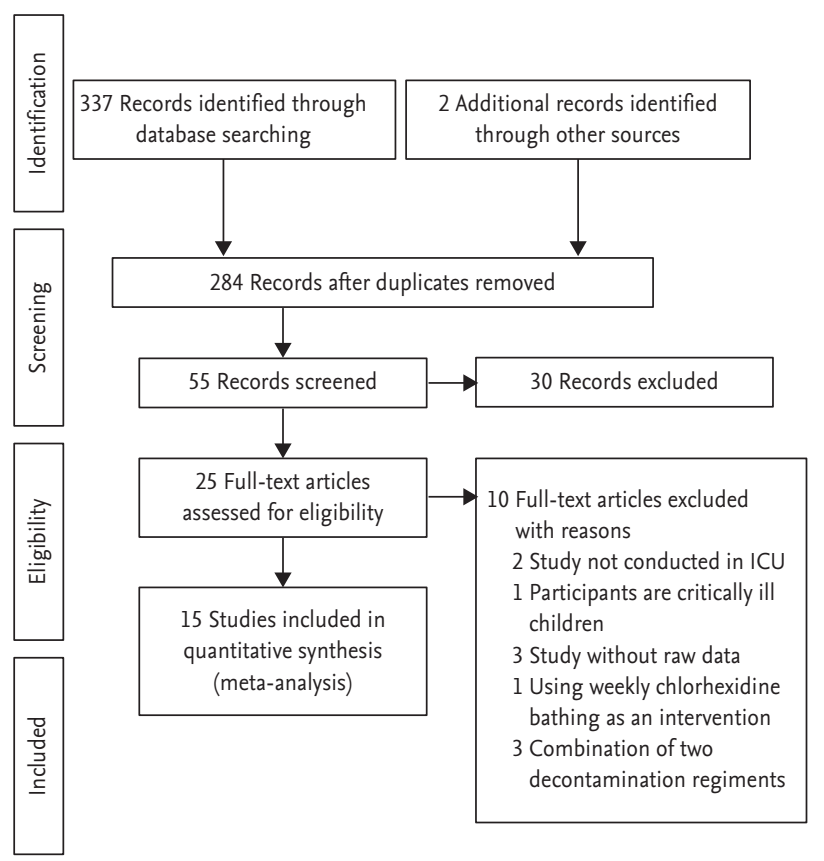

Figure 1. The process for studies selection. ICU, intensive care unit.

sessment for non-RCTs showed that the majority of the studies were rated with a score of more than 6 , except for two studies $[8,23]$.

\section{Primary outcomes}

\section{CRBSI}

Eleven studies [6-12,15,20-22] provided data on CHG bathing and CRBSI rates. Pooled results showed a significant difference between the CHG bathing arm and control arm on CRBSI rates (RR, 0.50; 95\% CI, 0.36 to $0.71 ; p<0.0001)$ and there was a moderate heterogeneity $\left(I^{2}=69 \% ; p=0.0004\right)$ (Fig. 2).

\section{CAUTI}

Data on CAUTI rates were extracted from seven of the included studies $[6,10,11,15,20,22,25]$. The results revealed that there was a significant difference between the two groups on CAUTI rates (RR, $0.68 ; 95 \% \mathrm{CI}$, 0.52 to $0.88 ; p$ $=0.004)$ and there was a low heterogeneity $\left(I^{2}=43 \% ; p=\right.$ 0.10) (Fig. 3).

\section{VAP}

Six studies $[6,10,11,15,20,22]$ assessed the effect of CHG bathing on reducing the incidence of VAP. The pooled 


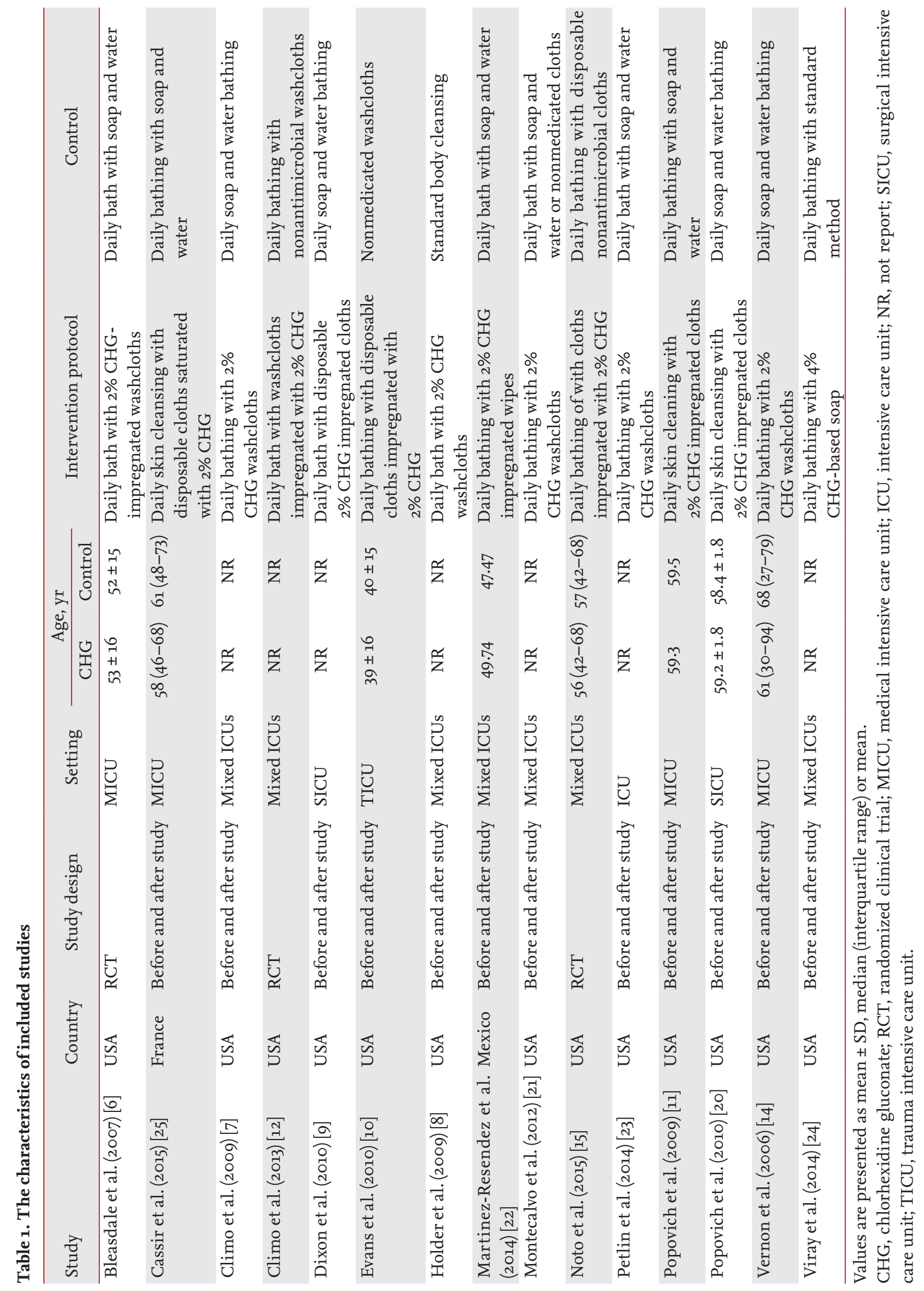




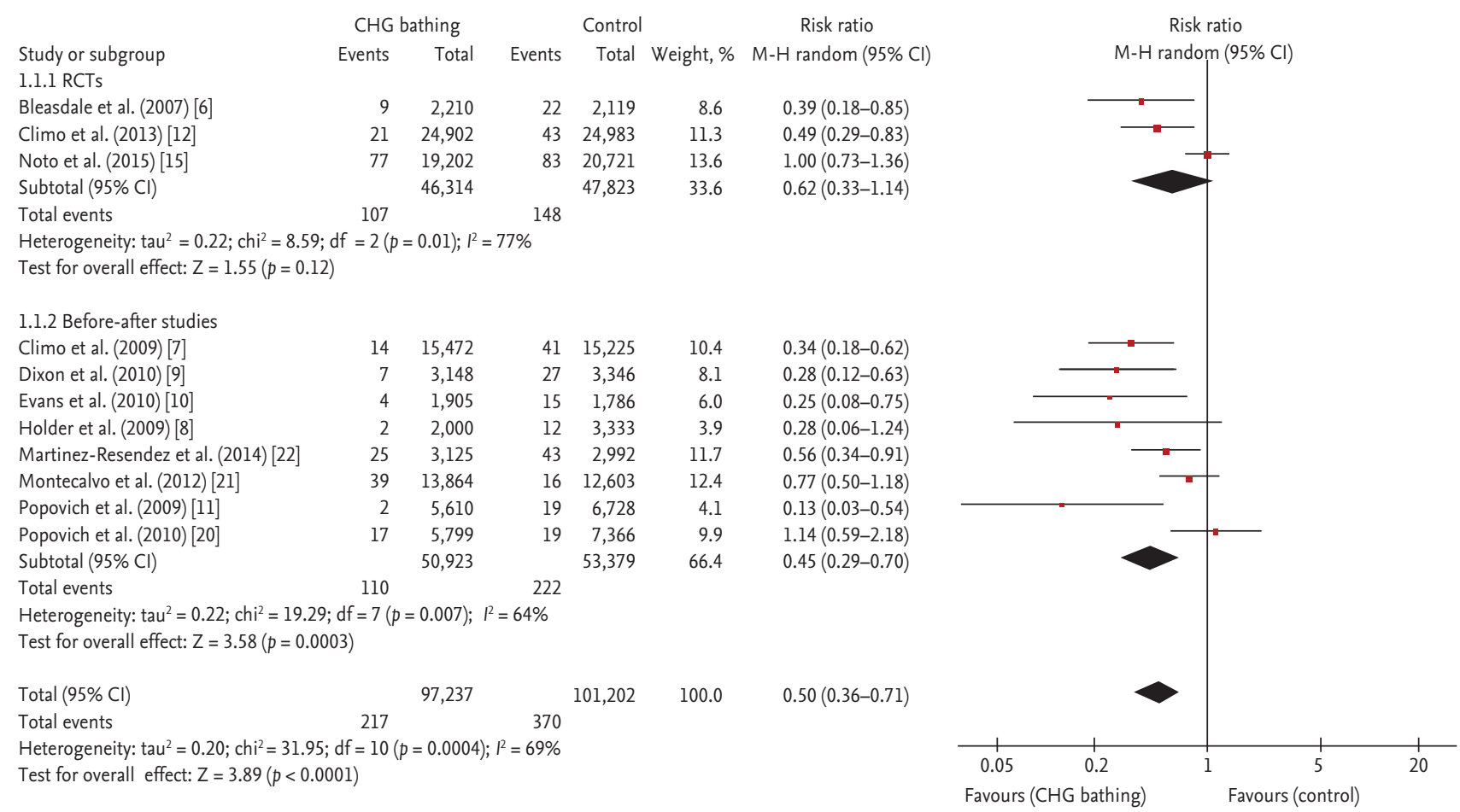

Figure 2. The effects of daily chlorhexidine gluconate (CHG) bathing in reducing catheter-related bloodstream infection. CI, confidence interval; RCT, randomized clinical trial; df, degrees of freedom; M-H, Mantel-Haenszel.

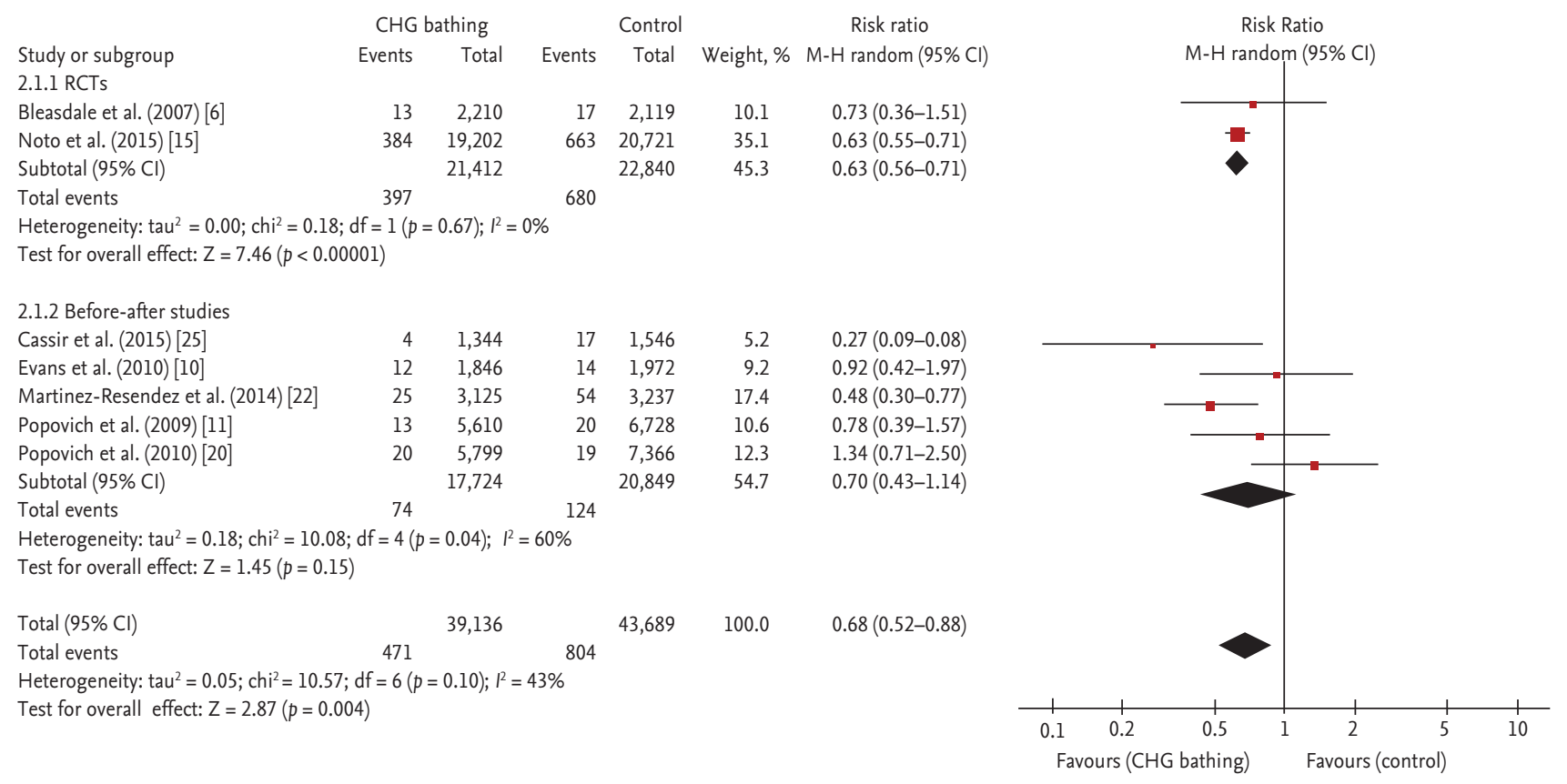

Figure 3. The effects of daily chlorhexidine gluconate (CHG) bathing in reducing catheter-associated urinary tract infection. CI, confidence interval; RCT, randomized clinical trial; df, degrees of freedom; M-H, Mantel-Haenszel. 

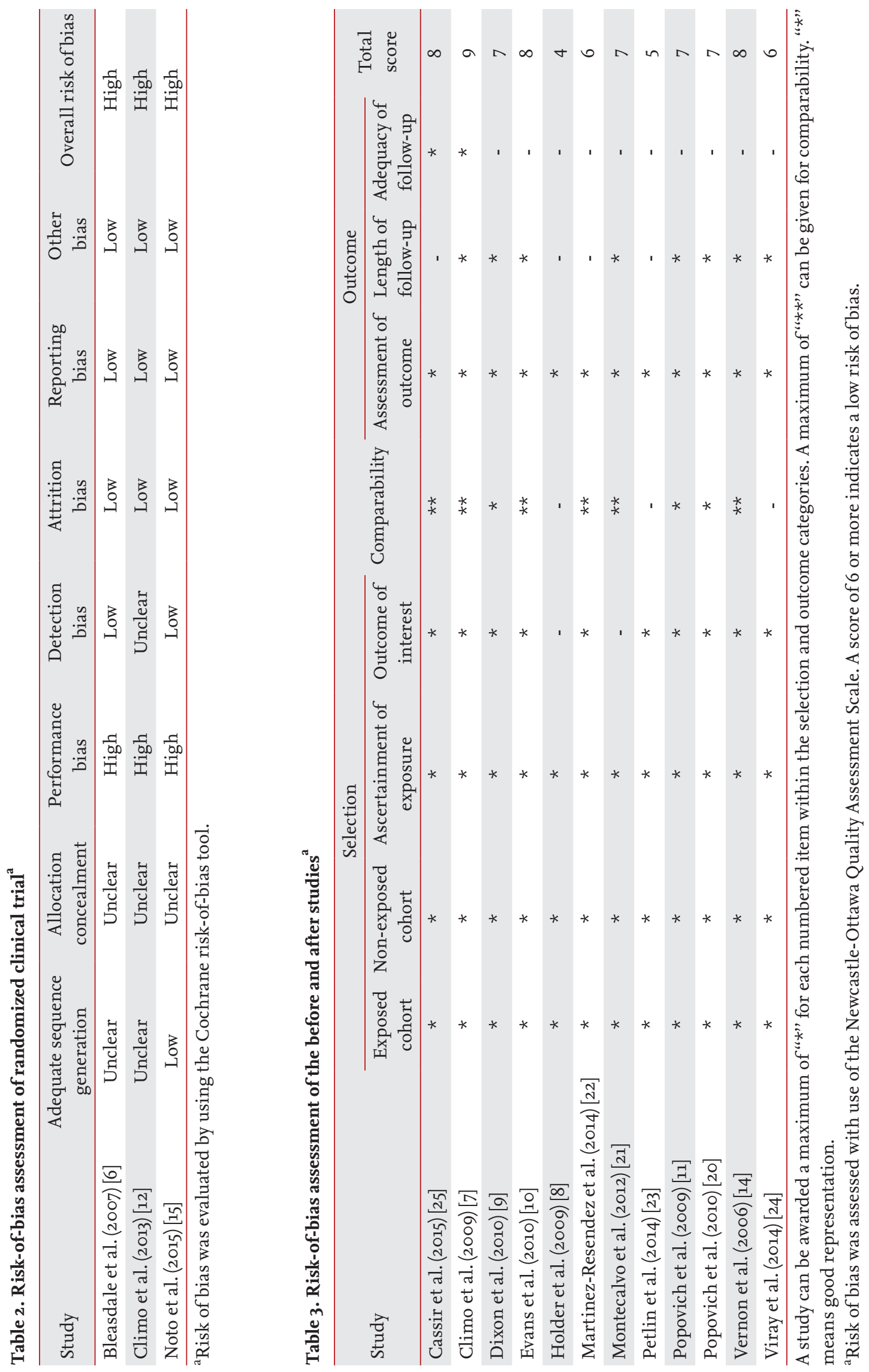


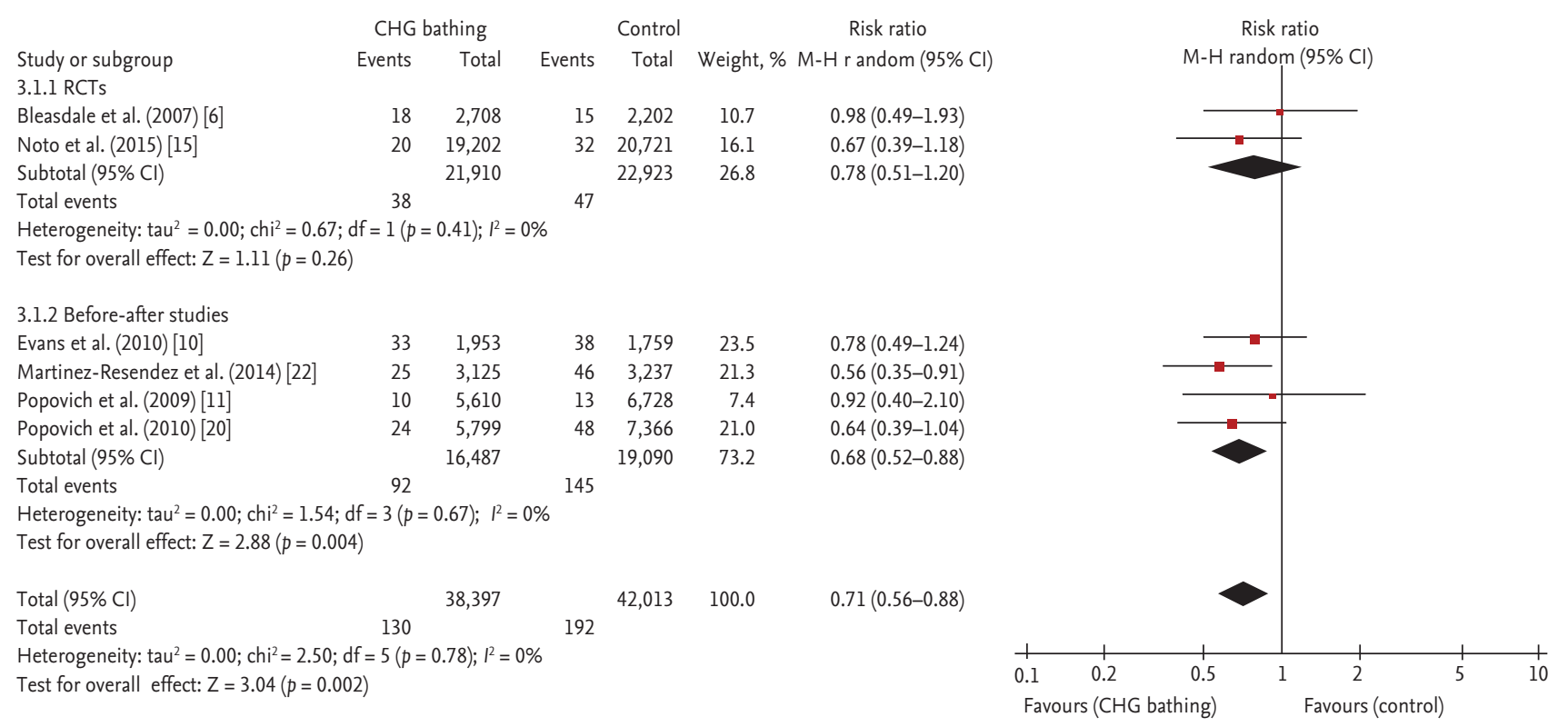

Figure 4. The effects of daily chlorhexidine gluconate (CHG) bathing in reducing ventilator-associated pneumonia. CI, confidence interval; RCT, randomized clinical trial; df, degrees of freedom; M-H, Mantel-Haenszel.

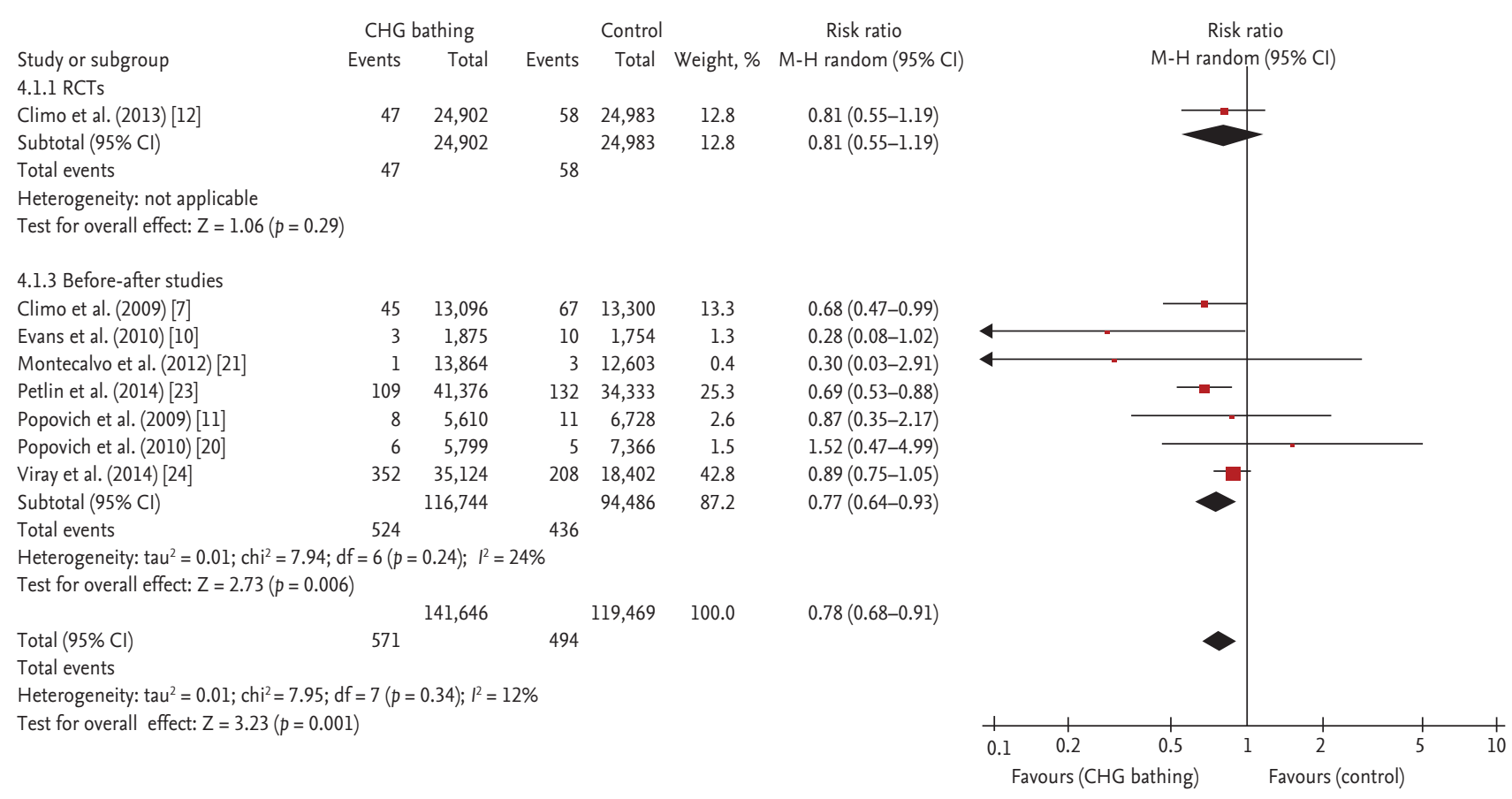

Figure 5. The effects of daily chlorhexidine gluconate (CHG) bathing in reducing methicillin-resistant Staphylococcus aureus acquisition. CI, confidence interval; RCT, randomized clinical trial; df, degrees of freedom; M-H, Mantel-Haenszel.

results showed that $\mathrm{CHG}$ bathing was strongly associated with a lower risk of VAP when compared with soap and water or other controls (RR, 0.71; 95\% CI, 0.56 to $0.88 ; p=0.002)$ and there was no heterogeneity $\left(I^{2}=0 \%\right.$; $p=0.78)($ Fig. 4).

MRSA acquisition

MRSA acquisition rates were available for eight stud- 


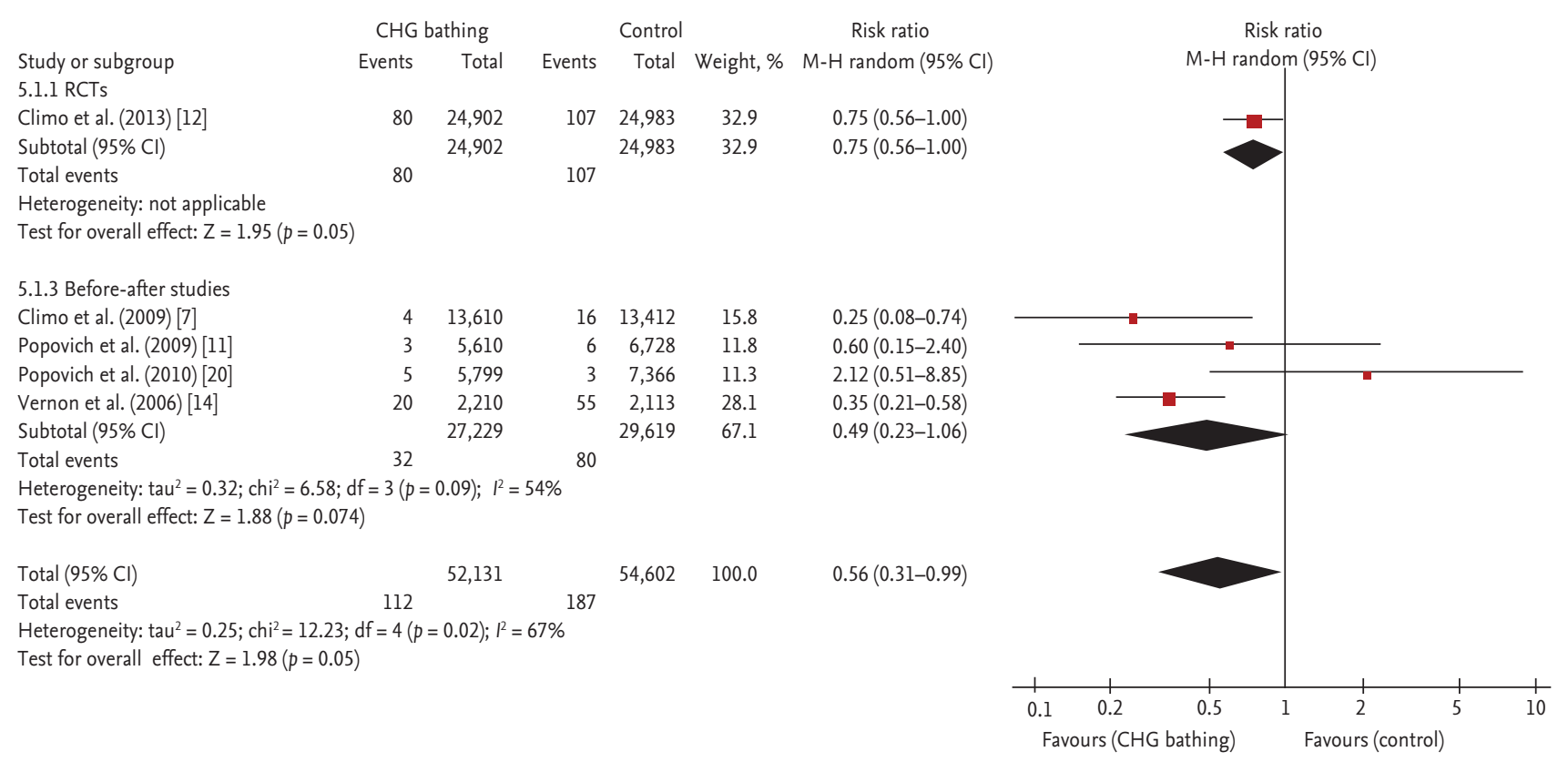

Figure 6. The effects of daily chlorhexidine gluconate (CHG) bathing in reducing vancomycin-resistant Enterococcus acquisition. CI, confidence interval; RCT, randomized clinical trial; df, degrees of freedom; M-H, Mantel-Haenszel.

ies, including one RCT [11] and seven before and after studies $[7,10,11,20,21,23,24]$, which showed a significant reduction in the risks of MRSA acquisition in the CHG bathing group (RR, $0.78 ; 95 \% \mathrm{CI}, 0.68$ to $0.91 ; p=0.001$ ) with a low heterogeneity $\left(I^{2}=12 \% ; p=0.34\right)$ (Fig. 5).

\section{VRE acquisition}

Five studies $[7,11,12,14,20]$ provided data on daily CHG bathing and VRE acquisition. The outcomes showed that daily CHG bathing was associated with decreased VRE acquisition (RR, 0.56; 95\% CI, 0.31 to $0.99 ; p=0.05$ ) and there was a moderate heterogeneity $\left(I^{2}=67 \%\right)$ (Fig. 6).

\section{Secondary outcomes}

\section{LOS}

Hospital LOS was reported in eight studies. However, four of studies did not provide the standard deviation of the outcome $[7,11,12,15]$. Pooled results revealed that there was no significant differences between the two groups on LOS (WMD, -0.27 ; 95\% CI, -0.68 to $0.14 ; p=$ $0.20)$ using the random-effects model $\left(I^{2}=44 \%\right)$.

Overall hospital mortality

Four studies $[10,15,22,25]$ involving 10,882 patients evalu- ated the effects of daily CHG bathing on overall hospital mortality. Outcomes showed that daily CHG bathing was associated with less hospital mortality (10.16\% vs. 11.45\%; RR, 0.88 ; $95 \%$ CI, 0.79 to $0.97 ; p=0.01$ ).

\section{Adverse events}

Only three studies evaluated adverse effects during the CHG bathing period. Bleasdale et al. [6] reported that three subjects were excluded from the CHG arm after developing rashes; however, it was ultimately determined not to be due to CHG. Evans et al. [10] witnessed two rashes that prevented continued use of CHG, both of which were caused by antibiotic therapy and resolved without intervention. Petlin et al. [23] did not find patient reports of skin irritation during the study.

\section{DISCUSSION}

This further meta-analysis demonstrated that daily CHG bathing had an overwhelming effect on decreasing the rates of the composite primary outcomes, including CRBSI, CAUTI, VAP, and acquisition of MRSA and VRE. However, there was no sufficient evidence to support that the use of daily CHG bathing can reduce hospi- 
tal LOS. In this meta-analysis, only studies conducted in adult ICUs were included; therefore, the results are not generalizable to hospitalized children. Fortunately, one well-designed trial can be a useful supplement for this specific population [26]. In this cluster-randomized, crossover study, the authors found that the incidence of bacterium was $36 \%$ lower among patients receiving daily CHG bathing compared with patients receiving standard bathing practices (3.28 vs. 4.93 per 1,000 days; adjusted incidence rate ratio, $0.64 ; 95 \% \mathrm{CI}, 0.42$ to 0.98 ) and there were no significant differences in the incidence of adverse events.

We found that there was a significant reduction CRBSI rates and the acquisition of MDROs when either $2 \%$ CHG-impregnated washcloths or CHG bathing were used, which is in accordance with previous systematic reviews [27,28]. To date, CHG bathing has been mainly employed in critical care settings; a limited number of studies were conducted in hospital-wide settings. One study conducted in a long-term acute care hospital reported that daily CHG baths can result in a net reduction of $99 \%$ in central venous CRBSI rates [29]. Another study performed in a general medical hospital provided strong evidence that daily bathing with CHG was associated with a $64 \%$ reduced risk of developing MRSA and VRE HAIs (hazard ratio, 0.36; 95\% CI, 0.2 to $0.8 ; p=0.01$ ) [30].

To our knowledge, this is the first study to evaluate the efficacy of daily CHG bathing on preventing VAP and CAUTI. Our findings suggest that daily CHG bathing will reduce the risk of VAP (RR, 0.71; 95\% CI, 0.56 to 0.88 ) and CAUTI (RR, 0.68 ; $95 \% \mathrm{CI}, 0.52$ to 0.88 ) in ICU settings. VAP is one of the most common HAIs in ICUs and occurs in $8 \%$ to $28 \%$ of patients receiving mechanical ventilation [31]. Aspiration of oropharyngeal pathogens into the lower respiratory tract is considered the major mechanism for the development of VAP [32]. Therefore, many strategies have been used to reduce bacteria in the oral cavity of patients who are mechanically ventilated [33]. Routine oral care with CHG has become the standard of care for patients receiving mechanical ventilation in most hospitals [34]. However, a recent meta-analysis [35] involving 16 studies showed that oral care with CHG did not decrease VAP risk in non-cardiac surgery patients and provided no additional benefits for patient-centered outcomes in either cardiac surgery or non-cardiac surgery patients. The relevant guidelines or recommendations about this practice may need to be reevaluated. In contrast, our study demonstrated that daily CHG bathing can significantly decrease the risk of VAP development. Several aspects probably contribute to this difference. First is the different methods of prevention (oral care vs. bathing) employed in both studies. Second is that the mean duration of mechanical ventilation in this study was shorter than in the study conducted by Klompas et al. [35], which may lead to an underestimation of VAP incidence.

Multiple guidelines to prevent CAUTI have been released $[36,37]$. Similar to CRBSI, many CAUTI prevention strategies have been bundled into a composite of several interventions, such as inserting catheters using aseptic technique and sterile equipment, hand hygiene, standard precautions, and so on. However, evidence for daily CHG bathing as routine care for preventing CAUTI is scant. Findings from our study provide more support for healthcare providers to use this approach. Previous culture-based analyses showed that hospitalized patients had a higher risk of skin colonization with gram-negative bacteria, particularly in the perineal area [38]. In the ICU, Escherichia coli accounts for approximately $18 \%$ to $26 \%$ of CAUTIIs [39]. Recently, a study assessed the effect of daily CHG bathing on skin microbiota [40]. The results showed that daily CHG bathing is associated with a reduction in gram-negative bacteria colonization together with substantial skin microbiota shifts. These findings may be a possible explanation for why daily CHG bathing can prevent CAUTI in our study.

Although this meta-analysis provides some evidence that daily CHG bathing can effectively prevent HAIs, some limitations should also be concerned. One is that only three eligible RCTs were included in this study. Therefore, the conclusions must be interpreted with caution. Second is that the overall quality of the included studies was low. None of the included RCTs used the double-blinding method, which may result in performance bias and detection bias. Third is that the included studies did not adequately evaluate the long-term effects of CHG bathing on overall mortality and adverse events, which are important outcomes for critically ill patients in ICUs.

In conclusion, this meta-analysis suggests that daily CHG bathing is significantly associated with lower risk of CRBSI, CAUTI, VAP, and acquisition of MRSA and 
VRE in ICU. However, more large sample size studies with long-term follow-up are needed to confirm and update these findings.

\section{KEY MESSAGE}

1. Daily chlorhexidine gluconate (CHG) bathing can significantly decrease the risk of healthcare-associated infections development in intensive care units, when compared with standard care.

2. Daily CHG bathing can reduce the overall hospital mortality. However, there is no influential on hospital length of stay.

3. Majority studies included in this meta-analysis were quasi-experimental studies, more well-designed randomized controlled trials are needed to confirm these findings.

\section{Conflict of interest}

No potential conflict of interest relevant to this article was reported.

\section{Acknowledgments}

All authors wish to thank the professor Jian-Rong, Zhou for her useful suggestions.

\section{REFERENCES}

1. Magill SS, Edwards JR, Bamberg W, et al. Multistate point-prevalence survey of health care-associated infections. N Engl J Med 2014;370:1198-1208.

2. Bonten MJ. Healthcare epidemiology. Ventilator-associated pneumonia: preventing the inevitable. Clin Infect Dis 2011;52:115-121.

3. Roberts RR, Scott RD 2nd, Hota B, et al. Costs attributable to healthcare-acquired infection in hospitalized adults and a comparison of economic methods. Med Care 2010;48:1026-1035.

4. Lim KS, Kam PC. Chlorhexidine: pharmacology and clinical applications. Anaesth Intensive Care 2008;36:502-512.

5. Popovich KJ, Lyles R, Hayes R, et al. Relationship between chlorhexidine gluconate skin concentration and microbial density on the skin of critically ill patients bathed daily with chlorhexidine gluconate. Infect Control Hosp Epidemiol 2012;33:889-896.

6. Bleasdale SC, Trick WE, Gonzalez IM, Lyles RD, Hayden MK, Weinstein RA. Effectiveness of chlorhexidine bathing to reduce catheter-associated bloodstream infections in medical intensive care unit patients. Arch Intern Med 2007;167:2073-2079.

7. Climo MW, Sepkowitz KA, Zuccotti G, et al. The effect of daily bathing with chlorhexidine on the acquisition of methicillin-resistant Staphylococcus aureus, vancomycin-resistant Enterococcus, and healthcare-associated bloodstream infections: results of a quasi-experimental multicenter trial. Crit Care Med 2009;37:1858-1865.

8. Holder C, Zellinger M. Daily bathing with chlorhexidine in the ICU to prevent central line-associated bloodstream infections. J Clin Outcomes Manag 2009;16:509-513.

9. Dixon JM, Carver RL. Daily chlorohexidine gluconate bathing with impregnated cloths results in statistically significant reduction in central line-associated bloodstream infections. Am J Infect Control 2010;38:817-821.

10. Evans HL, Dellit TH, Chan J, Nathens AB, Maier RV, Cuschieri J. Effect of chlorhexidine whole-body bathing on hospital-acquired infections among trauma patients. Arch Surg 2010;145:240-246.

11. Popovich KJ, Hota B, Hayes R, Weinstein RA, Hayden MK. Effectiveness of routine patient cleansing with chlorhexidine gluconate for infection prevention in the medical intensive care unit. Infect Control Hosp Epidemiol 2009;30:959-963.

12. Climo MW, Yokoe DS, Warren DK, et al. Effect of daily chlorhexidine bathing on hospital-acquired infection. $\mathrm{N}$ Engl J Med 2013;368:533-542.

13. Srinivas A, Kaman L, Raj P, et al. Comparison of the efficacy of chlorhexidine gluconate versus povidone iodine as preoperative skin preparation for the prevention of surgical site infections in clean-contaminated upper abdominal surgeries. Surg Today 2015;45:1378-1384.

14. Vernon MO, Hayden MK, Trick WE, et al. Chlorhexidine gluconate to cleanse patients in a medical intensive care unit: the effectiveness of source control to reduce the bioburden of vancomycin-resistant enterococci. Arch Intern Med 2006;166:306-312.

15. Noto MJ, Domenico HJ, Byrne DW, et al. Chlorhexidine bathing and health care-associated infections: a randomized clinical trial. JAMA 2015;313:369-378.

16. Moher D, Liberati A, Tetzlaff J, Altman DG; PRISMA 
Group. Preferred reporting items for systematic reviews and meta-analyses: the PRISMA statement. Ann Intern Med 2009;151:264-269.

17. Higgins JP, Altman DG, Gotzsche PC, et al. The Cochrane Collaboration's tool for assessing risk of bias in randomised trials. BMJ 2011;343:d5928.

18. Wells GA, Shea B, O'Connell D, et al. The Newcastle-Ottawa Scale (NOS) for assessing the quality of nonrandomised studies in meta-analyses [Internet]. Ottawa (ON): Ottawa Hospital Research Institute, c2014 [cited 2016 Jan 26]. Available from: http://www.ohri.ca/programs/clinical_ epidemiology/oxford.asp.

19. Higgins JP, Thompson SG, Deeks JJ, Altman DG. Measuring inconsistency in meta-analyses. BMJ 2003;327:557-560.

20. Popovich KJ, Hota B, Hayes R, Weinstein RA, Hayden MK. Daily skin cleansing with chlorhexidine did not reduce the rate of central-line associated bloodstream infection in a surgical intensive care unit. Intensive Care Med 2010;36:854-858.

21. Montecalvo MA, McKenna D, Yarrish R, et al. Chlorhexidine bathing to reduce central venous catheter-associated bloodstream infection: impact and sustainability. Am J Med 2012;125:505-511.

22. Martinez-Resendez MF, Garza-Gonzalez E, Mendoza-Olazaran S, et al. Impact of daily chlorhexidine baths and hand hygiene compliance on nosocomial infection rates in critically ill patients. Am J Infect Control 2014;42:713-717.

23. Petlin A, Schallom M, Prentice D, et al. Chlorhexidine gluconate bathing to reduce methicillin-resistant Staphylococcus aureus acquisition. Crit Care Nurse 2014;34:17-25.

24. Viray MA, Morley JC, Coopersmith CM, Kollef MH, Fraser VJ, Warren DK. Daily bathing with chlorhexidine-based soap and the prevention of Staphylococcus aureus transmission and infection. Infect Control Hosp Epidemiol 2014;35:243-250.

25. Cassir N, Thomas G, Hraiech S, et al. Chlorhexidine daily bathing: impact on health care-associated infections caused by gram-negative bacteria. Am J Infect Control 2015;43:640-643.

26. Milstone AM, Elward A, Song X, et al. Daily chlorhexidine bathing to reduce bacteraemia in critically ill children: a multicentre, cluster-randomised, crossover trial. Lancet 2013;381:1099-1106.

27. O'Horo JC, Silva GL, Munoz-Price LS, Safdar N. The efficacy of daily bathing with chlorhexidine for reducing healthcare-associated bloodstream infections: a meta-analysis. Infect Control Hosp Epidemiol 2012;33:257267.

28. Derde LP, Dautzenberg MJ, Bonten MJ. Chlorhexidine body washing to control antimicrobial-resistant bacteria in intensive care units: a systematic review. Intensive Care Med 2012;38:931-939.

29. Munoz-Price LS, Hota B, Stemer A, Weinstein RA. Prevention of bloodstream infections by use of daily chlorhexidine baths for patients at a long-term acute care hospital. Infect Control Hosp Epidemiol 2009;30:1031-1035.

30. Kassakian SZ, Mermel LA, Jefferson JA, Parenteau SL, Machan JT. Impact of chlorhexidine bathing on hospital-acquired infections among general medical patients. Infect Control Hosp Epidemiol 2011;32:238-243.

31. Chastre J, Fagon JY. Ventilator-associated pneumonia. Am J Respir Crit Care Med 2002;165:867-903.

32. Safdar N, Crnich CJ, Maki DG. The pathogenesis of ventilator-associated pneumonia: its relevance to developing effective strategies for prevention. Respir Care 2005:50:725-739.

33. Shi Z, Xie H, Wang $\mathrm{P}$, et al. Oral hygiene care for critically ill patients to prevent ventilator-associated pneumonia. Cochrane Database Syst Rev 2013;8:CDoo8367.

34. Lambert ML, Palomar M, Agodi A, et al. Prevention of ventilator-associated pneumonia in intensive care units: an international online survey. Antimicrob Resist Infect Control 2013;2:9.

35. Klompas M, Speck K, Howell MD, Greene LR, Berenholtz SM. Reappraisal of routine oral care with chlorhexidine gluconate for patients receiving mechanical ventilation: systematic review and meta-analysis. JAMA Intern Med 2014;174:751-761.

36. Gould CV, Umscheid CA, Agarwal RK, Kuntz G, Pegues DA; Healthcare Infection Control Practices Advisory Committee. Guideline for prevention of catheter-associated urinary tract infections 2009. Infect Control Hosp Epidemiol 2010;31:319-326.

37. Hooton TM, Bradley SF, Cardenas DD, et al. Diagnosis, prevention, and treatment of catheter-associated urinary tract infection in adults: 2009 International Clinical Practice Guidelines from the Infectious Diseases Society of America. Clin Infect Dis 2010;50:625-663.

38. Seifert H, Dijkshoorn L, Gerner-Smidt P, Pelzer N, Tjernberg I, Vaneechoutte M. Distribution of Acinetobacter species on human skin: comparison of phenotypic and 
genotypic identification methods. J Clin Microbiol 1997;35:2819-2825.

39. Shuman EK, Chenoweth CE. Recognition and prevention of healthcare-associated urinary tract infections in the intensive care unit. Crit Care Med 2010;38(8 Suppl):S373-S379.
40. Cassir N, Papazian L, Fournier PE, Raoult D, La Scola B. Insights into bacterial colonization of intensive care patients' skin: the effect of chlorhexidine daily bathing. Eur J Clin Microbiol Infect Dis 2015;34:999-1004. 